\title{
La comprensión de textos especializados. Un estudio polifónico- argumentativo sobre las dificultades de lectura en los estudios de formación docente en la Argentina
}

The comprehension of specialized texts. A polyphonic argumentative study on reading difficulties at a teacher training program in Argentina

\section{Volumen 17, Número 3 \\ Setiembre-Diciembre}

pp. 1-22

\section{Este número se publica el $1^{\circ}$ de setiembre de 2017 \\ DOI: https://doi.org/10.15517/aie.v17i3.29570}

\section{Carolina Tosi}

Revista indizada en REDALYC, SCIELO

Revista distribuida en las bases de datos:

LATINDEX, DOAJ, REDIB, IRESIE, CLASE, DIALNET, SHERPA/ROMEO, QUALIS-CAPES, MIAR

Revista registrada en los directorios:

ULRICH'S, REDIE, RINACE, OEI, MAESTROTECA, PREAL, CLACSO 


\title{
La comprensión de textos especializados. Un estudio polifónico- argumentativo sobre las dificultades de lectura en los estudios de formación docente en la Argentina
}

\author{
The comprehension of specialized texts. A polyphonic argumentative study on reading \\ difficulties at a teacher training program in Argentina
}

\begin{abstract}
Carolina Tosi ${ }^{1}$
Resumen. Diversas investigaciones lingüísticas sostienen que los problemas de comprensión lectora que manifiestan los estudiantes de la educación superior responden a la falta de desarrollo temprano de las habilidades letradas vinculadas con los textos de estudio. Ubicado dentro esa perspectiva, el presente artículo se encarga de mostrar que las principales dificultades de lectura académica presentadas por el estudiantado de nivel terciario se relacionan con la inadecuación a la práctica lectora de textos especializados, así como con la escasa destreza en la reflexión metalingüística. Para llevar a cabo la investigación, se trabajó con una población de estudiantes ingresantes a un profesorado de Educación Primaria e Inicial, en la Ciudad de Buenos Aires (Argentina), y se implementó la modalidad de investigador "participante-observador". El proceso de elaboración de los datos fue predominantemente cualitativo y el corpus de análisis se conformó con sesenta y tres producciones escritas, correspondientes a una actividad de comprensión lectora realizada sobre un texto argumentativo. Los resultados obtenidos manifiestan que los principales problemas de lectura detectados en el cuerpo estudiantil se vinculan con ciertos aspectos enunciativos y polifónicos de los textos. A lo largo del trabajo, se sistematizan las dificultades detectadas y se las asocia con las características de la configuración discursiva de los libros de texto, que se constituyen como el principal material de estudio de la escuela secundaria. Finalmente, el trabajo demuestra la importancia de poner en práctica "estrategias de facilitamiento" que fomenten la reflexión metalingüística del estudiantado, con el fin de mejorar su comprensión lectora.
\end{abstract}

Palabras clave: habilidades de lectura, lectura académica, formación docente, estudiantes universitarios.

\begin{abstract}
A number of linguistic studies have argued that the reading comprehension issues presented by students in higher education respond to the lack of early development of literacy skills associated with study texts. Within this perspective, this paper shows that the main difficulties of academic reading presented by tertiary students are related to the inadequacy of the reading practice of specialized texts as well as to the lack of skills in metalinguistic reflection. To carry out the research, we worked with a population of incoming students to a teacher training program for Primary and Initial Education at the City of Buenos Aires (Argentina) and implemented a participant-observer research methodology. The process of data elaboration was predominantly qualitative and the corpus of analysis was made up of sixty-three written productions, corresponding to an activity of reading comprehension carried out on an argumentative text. The obtained results show that the main issues involving reading skills are linked with certain enunciative and polyphonic aspects of the texts. Throughout the analysis, the difficulties detected are systematized and are associated with the characteristics of the discursive configuration of textbooks, which constitute the main study material of secondary schools. Finally, the study demonstrates the importance of implementing "facilitating strategies" that foster students' metalinguistic reflection in order to improve their reading comprehension.
\end{abstract}

Keywords: reading skills, academic reading, teacher training, university students

\footnotetext{
1 Profesora en la Universidad de Buenos Aires, Argentina. Investigadora asistente del Conicet (Consejo Nacional de Investigaciones Científicas y Técnicas), Argentina.
}

Dirección electrónica: carolinaltosi@gmail.com

Artículo recibido: 4 de octubre, 2016

Enviado a corrección: 15 de marzo, 2017

Aprobado: 5 de junio, 2017 


\section{Introducción}

En las últimas décadas, diversas investigaciones realizadas en la Argentina han puesto el acento en los problemas de lectura y escritura que manifiestan los estudiantes que ingresan a los estudios superiores (Arnoux, Nogueira y Silvestri, 2001, 2003 y 2006; Carlino, 2005; García Negroni y Hall, 2011; Hall y Marín, 2002, 2003 y 2011; Hall, 2007 y 2008; Pereira y Di Stéfano, 2001; entre otros). Entre tales investigaciones, se encuentra una línea de análisis que sostiene que los problemas de comprensión y escritura manifestados por los y las estudiantes responden a la falta de desarrollo temprano de las habilidades letradas vinculadas con los textos de estudio y que, lejos de relacionarse con el desconocimiento de requisitos lingüísticos y textuales, evidencian la inadecuación de los sujetos a la práctica científico-disciplinar (Carlino, 2005; García Negroni y Hall, 2011 y Hall, 2007 y 2008). Siguiendo tal perspectiva, el presente trabajo se propone, por un lado, indagar algunas de las dificultades que el estudiantado de un instituto de formación docente — de la Ciudad de Buenos Aires, Argentina- manifiesta en el abordaje de discursos especializados y, por el otro, plantear la necesidad de poner en práctica "estrategias de facilitamiento" — concepto definido en Tosi (2015), en el marco de una investigación sobre libros de texto desarrollada desde la teoría de la polifonía enunciativa (Ducrot, 2001) —, que promuevan la reflexión metalingüística y, por ende, la mejora en la comprensión de los textos.

Partimos del supuesto de que los problemas advertidos en el estudiantado, relacionados principalmente con la dificultad de reconocer la dimensión polémica y argumentativa de los materiales de estudio (Arnoux, Nogueira y Silvestri, 2001, 2003 y 2006), pueden encontrar una explicación en las características de los libros de texto que frecuentan en el nivel educativo anterior —la escuela secundaria—. Según se analizó en Tosi (2015), los libros de texto presentan "modos de decir pedagógico" funcionan como "mecanismos de simplificación" al atenuar los rasgos argumentativos complejos y los procedimientos de alteridad $^{2}$. Dado que los libros de texto suelen ser el primer contacto que tienen los y las estudiantes con los materiales de estudio, es factible sostener que el análisis y reflexión

\footnotetext{
2 En diversos trabajos hemos analizado que el ocultamiento del carácter argumentativo y polifónico de los libros de texto de nivel secundario. Por ejemplo, en Tosi (2015) se detectó una serie de rasgos enunciativos y argumentativos que se manifiestan con frecuencia y regularidad en todas las disciplinas de los libros de texto que denominamos "modos de decir pedagógico" y funcionan como mecanismos que buscan simplificar y allanar el aprendizaje. Entre ellos distinguimos: 1) la espacialización del saber, 2) la construcción del locutor-autor como regulador del sentido a través de la primera persona del plural con valor condescendiente, 3) la modalización autonímica (uso de tipografía espacial y comillas pedagógicas), y 4) una serie de recursos dispuestos para la construcción de la definición, como las falsas consecuencias de denominación, las cláusulas condicionales de denominación y la reformulación parafrástica de denominación.
} 
sobre las propiedades lingüísticas pueden constituir una herramienta imprescindible para enfrentar con éxito el abordaje de textos especializados, y promover la alfabetización académica.

Si bien, algunos autores plantean que las profundas dificultades que el estudiantado de los niveles superiores muestra tienen pocas posibilidades de revertirse (Johns y Swales, 2002). Nosotros, por el contrario, consideramos que un trabajo de reflexión metalingüística sostenido puede acarrear enormes mejoras en la comprensión lectora. En efecto, tal como sostienen Hall y Marín: "Creemos que ayudar a los alumnos a comprender e interpretar no significa 'facilitar' los textos, sino enseñar a procesar los textos más difíciles, porque eso es democratizar el acceso al saber" (2003, p. 09).

Como corpus específico de trabajo, examinamos las producciones generadas en una experiencia didáctica llevada a cabo en un taller de discurso académico destinado a ingresantes de un Profesorado de Educación Inicial y Primaria, y las complementamos con entrevistas escritas individuales.

En lo que sigue, primero, se presentan algunas aproximaciones teóricas y la metodología propuesta. En segundo término, se explica la experiencia del taller, se puntualizan las dificultades que manifestó el cuerpo estudiantil en la comprensión de un texto argumentativo, para luego vincularlas con las propiedades de los textos de estudio del nivel secundario. Seguidamente, se plantea la relevancia de aplicar "estrategias de facilitamiento", y por último, se enuncian las conclusiones.

\section{Aproximaciones teóricas sobre la lectura de textos académicos}

Es preciso recalcar que para encarar esta investigación se tomaron como base una serie de investigaciones producidas en la Argentina que atienden a la problemática específica del déficit de comprensión lectora manifestada por estudiantes ingresantes a los estudios superiores en dicho país. Entre ellos se destacan los trabajos de Pereira y Di Stéfano (2001); Arnoux, Nogueira y Silvestri (2001, 2003 y 2006) y Carlino (2005). Las autoras abordan la producción de textos académicos por parte de estudiantes terciarios y universitarios, y centran su interés en la dimensión argumentativa de los textos con los que ellos se enfrentan. Por un lado, estos enfoques coinciden en que la mayor dificultad que presentan los manuales universitarios ${ }^{3}$ reside en el ocultamiento del carácter argumentativo,

\footnotetext{
${ }^{3}$ Las autoras abordan los manuales universitarios que se utilizan especialmente en los cursos de ingreso o en los primeros años de las carreras de grado de universidades nacionales.
} 
el cual se constituye como una característica de los textos que comunican conocimiento (Pereira y Di Stéfano, 2001). De este modo, tales materiales no iniciarían verdaderamente a los estudiantes en la alfabetización académica.

Por otro lado, parte de los estudios referidos hacen foco en que, debido a que el grupo de ingresantes no está habituado a leer textos académicos, carece de los modos de pensamiento, explicación y argumentación, así como de representaciones comunes a las de los autores científicos. En este sentido, Carlino (2005) destaca la falta de códigos compartidos entre autor y lector en los textos académicos y que, según su hipótesis, da origen a problemas de comprensión. Para Carlino, los problemas de lectura "no se deben a que los estudiantes carecen de una habilidad o técnica elemental y generalizable, sino que al ingresar a los estudios superiores se ven enfrentados a nuevas culturas escritas" (2005, p. 85).

Respecto de los estudiantes de nivel superior, Arnoux, Nogueira y Silvestri (2001 y 2003) señalan que estos muestran dificultades no solo para reconocer las voces en los textos argumentativos y establecer relaciones entre ellas, sino también para aunar esas voces y relaciones en una representación textual integradora (2003, p. 179). En un trabajo posterior de 2006, dichas autoras se encargan de analizar específicamente las competencias discursivas de futuros docentes de nivel primario, relativas a la comprensión lectora y la producción de textos teóricos expositivo-explicativos. Su estudio da cuenta de las escasas habilidades de escritura de los y las estudiantes, evidenciadas en las respuestas altamente reproductivas respecto de la fuente; la construcción de textos poco articulados; la baja cohesión y una insuficiente autonomía explicativa. Teniendo en cuenta este antecedente, el presente trabajo aborda la comprensión lectora de estudiantes de formación docente, pero en otro tipo de textos, los argumentativos, con el objetivo de realizar una serie de observaciones acerca de los aspectos que debe considerar una pedagogía de la lectura en la formación docente, porque "si queremos transformar la educación en nuestros países debemos considerar seriamente la formación de los maestros" (Arnoux, Nogueira y Silvestri, 2006, p. 18).

Por otra parte, vale mencionar que García Negroni y Hall (2011) sistematizan las dificultades de la escritura de los estudiantes universitarios (i.e. ambigüedad en la presentación de las voces responsables de los enunciados, atemporalidad que cae en el anacronismo, contradicciones y falta de consistencia en las relaciones lógicas y argumentativas) y las caracterizan como una serie de "procedimientos inadecuados" en 
relación con su consistencia argumentativa. Denominados "distorsiones enunciativas", estos recursos corresponden a un intento fallido de imitación del modo de decir del discurso académico por parte del estudiantado. Ubicadas en esta misma corriente, Hall y Marín (2011) identifican las nominalizaciones, participios adjetivales, negaciones metadiscursivas y construcciones concesivas, causales y consecutivas, entre otras, como los principales escollos interpretativos en el decir académico-pedagógico (manuales universitarios).

En relación con ello, según se demostró en un trabajo previo (Tosi, 2015), las dificultades que genera el material pedagógico de la escuela media exceden el plano conceptual, léxico o textual en los que suelen hacer hincapié los estudios más difundidos de las Ciencias de la Educación o de la didáctica disciplinar —como las que se centran en las dificultades que presentan la "transposición didáctica" (Chevallard, 1991) o los "contenidos protodisciplinares" (Litwin, 1997) o los que se focalizan en las secuencias textuales (Avendaño y Perrone, 2009; Lerner, 2001; Padilla 2010) o la lectura por objetivos (Solé, 1999)-.

Sobre esta base y según Arnoux, Nogueira y Silvestri (2001, 2003 y 2006), García Negroni y Hall (2011) y Hall y Marín (2011), nuestra investigación ratifica que los problemas advertidos en el estudiantado se vinculan sustancialmente con los aspectos enunciativos y polifónico-argumentativos presentes en los textos académicos, y agregamos que estos aspectos discursivos suelen ser neutralizados en los manuales escolares de secundario. Cabe recordar que, de acuerdo con la Polifonía Enunciativa (Ducrot, 2001), corriente surgida en los años ochenta que rechaza el supuesto de que la función necesaria del lenguaje sea representar la realidad, los discursos se constituyen como un complejo construido por una multiplicidad de voces y puntos de vista.

De ahí que pueda afirmarse que la polifonía y la argumentación son dos dimensiones constitutivas e indisociables del lenguaje. Sin embargo, los libros de texto que suelen emplearse en el nivel medio buscan atenuar la manifestación de ambas dimensiones mediante la apelación a diversos recursos microdiscursivos, como por ejemplo la no explicitación del discurso ajeno. Al priorizar la inclusión de textos sencillos y "llanos", soslayan las propiedades discursivas "complejas" de los materiales de estudio, con las que el estudiantado debería familiarizarse para lograr el acceso a la cultura escrita especializada. En consecuencia, los libros escolares simplifican los textos para auxiliar a sus destinatarios, pero no contribuyen a facilitar y promover la alfabetización académica (Tosi, 2015). 
Finalmente, es posible postular que las "nuevas culturas", mencionadas por Carlino (2005), a las que se enfrenta el cuerpo estudiantil en el nivel superior no solo vehiculizan una representación sobre el saber, diferente de la que presentan los textos de estudio que circulan en el nivel medio —a los que los estudiantes estarían más habituados-, y que implica también una forma distinta de leer y de comprender, sino también otra configuración microdiscursiva que les es ajena, extraña y hasta, en ocasiones, insoslayable.

\section{Metodología y objetivos}

Como ya se adelantó, este trabajo retoma los resultados de una investigación en proceso en la que se analizan los mecanismos microdiscursivos de los libros de texto de secundario de diferentes disciplinas. Se indaga en qué medida su formulación lingüística incide en el acceso del estudiantado a la lectura especializada (Tosi, 2015).

Los propósitos del presente trabajo son tres: a) detectar los principales problemas de comprensión que presenta un grupo de estudiantes en el nivel superior, b) establecer una relación entre estas dificultades y la configuración discursiva de los libros de texto, y c) demostrar la relevancia del planteo de "estrategias de facilitamiento" que fomenten la reflexión metalingüística con el fin de mejorar la comprensión lectora.

La experiencia que, en esta oportunidad, se exhibe fue llevada a cabo en el taller de "Escritura Académica", en el marco del "Espacio de Definición Institucional" de un Profesorado de Educación Primaria e Inicial ${ }^{4}$. Vale aclarar que estos profesorados en la Ciudad de Buenos Aires cuentan con un tramo que se denomina "Espacio de Definición Institucional" (EDI). Inscripto en los nuevos lineamientos político-nacionales de Formación Docente y Políticas Curriculares del Ministerio de Educación de la Ciudad Autónoma de Buenos Aires (Resolución № 6626/09), el plan de estudios contempla la existencia de diferentes "EDI" dentro del Campo de la Formación Específica del nivel, cuyo fin consiste en delinear recorridos formativos para los futuros docentes que respondan al ideario de cada institución y a las demandas específicas de su comunidad educativa. Los EDI ayudarían a definir, así, una identidad y un perfil institucional particular del futuro egresado.

En el proyecto de la institución que aquí se aborda, se definieron como EDI los talleres de discurso académico, en atención a los problemas de lectura y escritura que presentaban las personas ingresantes. El taller, cuya cursada se suele sugerir durante el primer año de la

\footnotetext{
${ }^{4}$ En la Escuela Normal Superior Nro. 4 - Instituto Nacional de Formación Docente, sita en el barrio de Caballito, en la Ciudad de Buenos Aires, Argentina. Es de administración estatal y depende del Gobierno de la Ciudad de Buenos Aires. Allí se forman futuros docentes de escuela de nivel inicial y primario.
} 
carrera - por ello, son ingresantes y en la mayoría recién egresadas del nivel secundario-, es cuatrimestral y tiene una frecuencia semanal de dos horas-cátedra (40 minutos cada una).

Este trabajo analiza una actividad de lectura que se realizó en la cuarta clase del taller en tres cursos diferentes a lo largo de un mismo cuatrimestre. El proceso de elaboración de los datos fue predominantemente de tipo cualitativo, en la medida en que los abordajes cualitativos se adecuan mejor a las investigaciones en grupos y segmentos delimitados (de Souza Minayo, 2009, p. 158). Para la conformación del corpus de análisis, se trabajó con un conjunto de técnicas de recolección de datos. Por un lado, en la primera clase, al estudiantado se le realizaron entrevistas semiestructuradas (de Souza Minayo, 2009, p. 158) escritas e individuales, con el propósito de relevar sus hábitos de lectura y averiguar sus experiencias en relación con los materiales de estudio. Esta herramienta de análisis prevé pocas preguntas y apunta a comprender los puntos de vista de los actores sociales como sujetos de la investigación (de Souza Minayo, 2009).

Si bien la entrevista consta de diez preguntas, para este trabajo nos centramos en abordar tres, debido a su relevancia para el análisis en cuestión, a saber:

1) ¿Qué material de estudio frecuentaba en el secundario?

2) ¿Qué lee actualmente?

3) ¿Qué diferencias principales observa entre el material de estudio del secundario y el del terciario?

Con respecto a 1), el $80 \%$ del estudiantado indicó que en el nivel secundario utilizaban libros de texto como material de estudio. Un 10\% señaló el uso de un corpus con material preparado por el mismo docente, aunque este solía incluir también fotocopias de capítulos o unidades temáticas de los libros de texto ${ }^{5}$. Solo un $10 \%$ indicó que utilizaban como material de estudio libros de la especialidad, o bien empleaban recursos digitales (como los del portal educ. $\left.a r^{6}\right)$.

En relación con 2), los estudiantes respondieron, en su gran mayoría, que actualmente leen el material de estudio del profesorado, así como libros de "novelas de amor" y autoayuda. Como se observa, los géneros elegidos "libremente" —es decir, que son leídos por elección propia, como los de literatura romántica y la autoayuda- están alejados del

\footnotetext{
${ }^{5}$ El $90 \%$ de los estudiantes proviene directamente del nivel secundario; solo un $10 \%$ realizó sus estudios en otra carrera.

${ }^{6}$ Se trata del portal oficial de la educación en la Argentina. Contiene un portal de contenidos educativos, un plan de capacitación docente y un plan de conectividad. Su acceso es libre y gratuito.
} 
ámbito académico. Ninguna de las personas estudiantes mencionó leer en sus ratos libres bibliografía especializada o propia de alguna de las materias del profesorado.

Si nos referimos a las respuesta de 3), casi la totalidad del cuerpo estudiantil coincidió en que los textos del terciario son más extensos, complejos y "oscuros" que los que leían en el secundario. En general, mencionaron la dificultad para encontrar las ideas principales, a causa de que en los textos académicos se "incluyen muchas citas", aparecen palabras muy "difíciles" y, además, no hay "palabras destacadas en negrita" como sí sucedía en los manuales escolares, estas auxiliaban la lectura ya que, de acuerdo con sus comentarios, ofrecían pistas de dónde se alojaban los tecnicismos o las ideas más importantes del texto.

Como evidencian las respuestas, los libros de texto del secundario parecen ser los materiales de estudio más usuales, y los alumnos hacen hincapié en que sus textos son "sencillos", breves y están muñidos de aspectos discursivos que simplifican la lectura, como las palabras destacadas en negritas y la escasez de voces ajenas explícitas en la explicación teórica.

De este modo, la información aportada por las entrevistas alentó nuestra hipótesis inicial: los libros de texto presentan una configuración microdiscursiva diferente de la académica y, efectivamente, se cimenta como el material de estudio de mayor frecuencia de uso en el secundario.

Por otro lado, vale destacar que en nuestra investigación se empleó la técnica de participante-observador (Duranti, 2000 y Gumperz, 1982). Luego de abordar diferentes textos narrativos, expositivos y argumentativos, y examinar sus características, en la cuarta clase del taller se le propuso al estudiantado un trabajo de comprensión lectora respecto de un texto argumentativo breve (el prólogo de un libro) de una temática de relevancia social la censura en la actualidad- (cf. Anexo, al final del trabajo). Se eligió trabajar con ese tipo de material pues las mayores dificultades de interpretación que manifestaron los y las estudiantes, en las clases previas, fueron ante textos argumentativos. Como ratifican diversas investigaciones (Arnoux, Nogueira y Silvestri, 2001, 2003 y Parodi Sweis, 1999), los textos argumentativos revelan una mayor complejidad enunciativa y polifónica que los narrativos y expositivos, y por ende, demandan un gran esfuerzo cognitivo por parte del estudiantado.

Las consigas que los estudiantes debían responder apuntaban a indagar sobre la hipótesis propuesta por el autor Néstor Ruiz (2000) y las técnicas argumentativas desarrolladas (conceptos explicados en las clases previas) (Anexo, al final del trabajo). 
Decidimos realizar solo dos preguntas para que sea un trabajo breve que pueda desarrollarse en las dos horas-cátedra de la clase; sin embargo, se consideró que estas fueran lo suficientemente relevantes como para abordar la comprensión textual en vinculación con el contenido textual y la estructura del texto argumentativo. La muestra final está conformada por un total de 63 producciones (18 perteneciente a un grupo, 22 a otro y 23 a un tercero).

Finalmente, cabe destacar que existieron tres variables de análisis. En primer lugar, la variable independiente, que supone el trabajo de la docente investigadora (observadoraparticipante) en la elaboración de estrategias de lectura (las llamadas "de facilitamiento"). En segundo lugar, las variables dependientes, que se relacionan con los efectos que se busca producir por el trabajo con la variable independiente. Aquí se detectó el avance en comprensión lectora de los y las estudiantes (a través de los cambios en estrategias de lectura manifestadas por ambos, la realización de entrevistas, etc.). Y, en tercer lugar, la variable asociada - se trata de un aspecto que puede influir en las variables dependientes pero que el investigador no controla-, que tiene que ver con las ideas manifiestas del estudiantado sobre materiales de lectura y la comprensión lectora.

\section{Resultados y análisis}

\subsection{Análisis de las producciones}

Como ya mencionamos, en las clases previas a la lectura del texto bajo análisis se indagaron diferentes textos argumentativos —incluso, algunos sobre el tema de censura- y se analizó su estructura mediante la indagación de sus características (hipótesis, componente polémico, refutación de contraargumentación, conclusiones, etc.) y mediante el abordaje de las técnicas retóricas utilizadas (citas de autoridad, refutación de citas, metáforas, comparaciones, etc. $)^{7}$.

Según puede observarse, el texto elegido para el ejercicio se organiza a partir de segmentos polémicos, y Ruiz parte de ciertos discursos previos que plantean diferentes conceptos de censura. Por medio de la evocación de esas otras voces y/o su refutación, el autor construye su propia noción de censura en la actualidad. De este modo, las voces ajenas y la dimensión polémica emergen a través de diferentes mecanismos microdiscursivos, que los estudiantes rara vez logran identificar. En efecto, al procesar las

\footnotetext{
${ }^{7}$ Para ello, se trabajó con el capítulo sobre argumentación del libro de Arnoux, Di Stéfano y Pereira (2002).
} 
respuestas del estudiantado, encontramos tres dificultades principales que pueden agruparse de la siguiente manera:

El estudiantado presentan problemas para:

- Encontrar la hipótesis del texto.

- Identificar las voces ajenas y sus funciones (marcos de análisis, puntos de vista, citas de autoridad, refutación, etc.).

- Descubrir la dimensión polémica/argumentativa del texto.

Respecto de la identificación de la hipótesis del texto, solo un $20 \%$ de las producciones esbozó el concepto de censura propuesto por Ruiz, que puede enunciarse de diversas formas, ya que la hipótesis no se formula explícitamente, sino que está implícita en el texto. A modo de ejemplo, mostramos las respuestas de cuatro estudiantes que lograron recuperar la idea planteada por el autor acerca de que la censura ha cambiado y que, en la actualidad, opera por exceso de información y unificación del (1 a 4). Incluso, en los ejemplos (1) y (4), observamos cómo dos estudiantes dan cuenta de la postura de la que disiente Ruiz, a partir de la cual plantea su tesis personal, vinculada con que la censura opera por saturación y acumulación de datos.

(1) Para Ruiz, hoy la censura no funciona suprimiendo, amputando, prohibiendo, cortando; actúa, por el contrario, por demasía, por acumulación. No permite asimilar ni pensar. Hay demasiado para consumir y, por lo tanto, no se percibe lo que falta.

(2) Ruiz propone un concepto nuevo de censura. Dice que el monopolio de la información unifica el mensaje y permite una "globalización" de la censura. Lo cierto es que la censura no ha desaparecido. Tan sólo ha cambiado de apariencia: se presenta por exceso y unificación de la información.

(3) Para el autor, la censura tradicionalmente entendida no existe, ya que no hay supresión de la información, muy por el contrario, lo que se observa es exceso de información.

(4) El autor no está de acuerdo con la postura que indica que la censura se basa en la prohibición. Dice que hay que eliminar tal definición y propone una nueva. Así Ruiz afirma que la saturación de información hace que los usuarios se vean con dificultad para asimilarla y por ende ahí es donde se produce la censura. 
Por el contrario, un $80 \%$ de estudiantes formuló otras hipótesis que, por lo general, refieren a las nociones de censura que el autor toma como punto de partida para elaborar su propia definición o que directamente refuta. A modo de ejemplo, presentamos algunas de las respuestas:

(5) El texto "La macdonalizción del pensamiento" habla acerca de la censura donde apunta a suprimir, prohibir, ocultar y esconder distintos hechos que suceden en la cotidianeidad.

(6) La hipótesis que sostiene el autor es que el discurso de la censura es un discurso que consiste esencialmente en prohibir los hechos u ocultarlos.

(7) Ferreyra propone que la censura es "formarse un juicio sobre una cosa, juzgar con sentido crítico", y en segundo lugar implica corrección o reprobación de una cosa.

(8) El autor propone que se ha macdonalizado el pensamiento.

Como es evidente, las respuestas (5) y (6) remiten a una concepción de censura que el autor refuta - presente al inicio del texto-, y que evoca como marco para su propia conceptualización. La referencia a ese discurso es realizada por Ruiz a partir de una expresión encabezada con un "se" impersonal, que puede remitir a la voz de la doxa, del saber general: "Se ha dicho que el discurso de la censura es un discurso que consiste, esencialmente, en amputar, suprimir, prohibir un cierto número o el conjunto de los hechos, ocultarlos, esconderlos". Respuestas de este tipo estarían mostrando que los y las estudiantes no logran identificar la voz ajena vehiculizada en el "se" impersonal y atribuyen dicha afirmación al autor del texto. De ahí que sea posible afirmar que la mayoría del estudiantado no puede realizar correctamente la atribución de este enunciado a su fuente enunciativa.

Sumado a ello, también representarían escollos para la comprensión, las expresiones formadas con infinitivos, como la frase siguiente en el texto ("Razonar de esta forma es creer que, dada la abundancia de información, que estamos en un universo donde los elementos son constantes") y ciertos conectores, como el adversativo pero, que introduce un refutación ("Pero hoy la censura no funciona mediante este principio") ${ }^{8}$. Puede sostenerse que la disposición de todos estos mecanismos microdiscursivos aumenta la dificultad para la

\footnotetext{
${ }^{8}$ Recordemos que el texto de este primer párrafo es: "Se ha dicho que el discurso de la censura es un discurso que consiste, esencialmente, en amputar, suprimir, prohibir un cierto número o el conjunto de los hechos, ocultarlos, esconderlos. Razonar de esta forma es creer que, dada la abundancia de información, estamos en un universo donde los elementos son constantes. Pero hoy la censura no funciona mediante este principio".
} 
comprensión en un lector inexperto, y por eso, se producen respuestas del tipo (5) y (6). En efecto, observamos que al cuerpo docente le cuesta interpretar los aspectos enunciativos y polifónico-argumentativos de los textos y distinguir la voz del enunciador de los discursos citados, así como percibir el valor de los conectores (como el valor refutativo del adversativo "pero") y la dimensión polémica.

Tales dificultades se relacionan con dos aspectos enunciativos y polifónicos inherentes a la estructura de los textos argumentativos. Como advirtieron Arnoux, Noguiera y Silvestri (2003), el estudiantado poco entrenado manifiesta, por un lado, la dificultad de integrar enunciados refutativos que confrontan dos posiciones, una de las cuales debe inferirse a partir de índices de modalidad negativa y, por el otro, la de reconocer la atribución de segmentos textuales a voces diferentes de las del autor.

Respecto de (7), el estudiante alude a la definición de diccionario que evoca "Ferreyra". Por un lado, se observa que consigna el nombre del autor del libro (Ferreyra, escrito incorrectamente, ya que se trata de Ferreira) en vez del responsable del prólogo (Ruiz), lo cual muestra, además, la confusión en la lectura de la fuente bibliográfica. Por otro, el estudiante atribuye la definición de diccionario de "censura" al autor, cuando en realidad este la reformula para crear una nueva acepción. Una vez más, el lector no logra percibir la dimensión polifónica y argumentativa del discurso. Finalmente, en el caso (8) el estudiante plantea como hipótesis el título del texto, sin explicar los alcances del concepto.

En relación con la segunda pregunta efectuada en el cuestionario, gran cantidad de informantes menciona que la narración sobre la censura en Roma y algunas citas avalan la postura del autor. Respecto de este último punto, el $75 \%$ de las respuestas muestra que las personas participantes solo identifican las voces ajenas que se presentan mediante discurso directo, aunque no siempre reconozcan su función o identifiquen su fuente. Por ejemplo, respecto de la cita de Pavese, a quien Ruiz utiliza como argumento de autoridad ("como diría Pavese"), el lector de (9) la interpreta, por el contrario, como un contraargumento que luego este refuta. Por su parte, si bien en (10) el estudiante reconoce la presencia de una voz ajena, que aparece entrecomillada, esta es atribuida a los romanos — que son mencionados después- y no al discurso lexicográfico al que, en realidad, corresponde.

(9) El autor discute con Pavese: Esta investigación intenta ser, como diría Cesare Pavese, "la auscultación de una perplejidad" llamada Argentina. Pero Ferreira lo niega y dice que se trata de la pesada tarea de vivir en una sociedad que se funde entre los silencios cómplices y la intolerancia. 
(10) En efecto, censurar es, según los romanos, "formarse un juicio sobre una cosa, juzgar con sentido crítico".

De esta forma, el análisis de las respuestas reveló que los discursos ajenos introducidos mediante mecanismos diferentes del discurso directo -como usos impersonales, nominalizaciones, etc.- son difícilmente advertidos por los y las estudiantes.

\subsection{Vínculos con el discurso de los libros de texto}

Como es evidente, los problemas detectados en el estudiantado pueden vincularse con las características de los textos escolares. Estos presentan, tal como ya se anunció, mecanismos de "simplificación". En Tosi (2015), se trabajó con un corpus de libros de texto de secundario y se detectó una serie de rasgos enunciativos y argumentativos que se manifestaban con frecuencia y regularidad en todas las disciplinas abordadas (Lengua y literatura, Ciencias Sociales y Ciencias Naturales). Si bien, se distinguieron los cuatro tipos de "modos de decir pedagógico" que ya mencionamos, aquí nos centramos en abordar aquel que se corresponde con la configuración discursiva: la "espacialización del saber". En efecto, el libro de texto contempla un espacio para el "saber disciplinar", es decir, el texto central teórico que, al eludir la explicitación de sus fuentes, aparece como si fuera objetivodesprovista de un sujeto enunciador- . De este modo, el locutor-autor busca mostrar que existe un saber escolar portador de una verdad unívoca. Este imaginario de referencialidad y neutralidad se consigue a partir de la puesta en foco de secuencias expositivo-explicativas centrales, a las que se las despoja de toda explicitación de discurso ajeno, y así se evita exhibir la dimensión argumentativa y polifónica.

Por otro lado, también se demostró en esa oportunidad que los libros de texto carecen de mecanismos de "facilitamiento" estimados como imprescindibles para la comprensión, ya que, según nuestra hipótesis, su aplicación puede promover la reflexión metalingüística y la desnaturalización de formulaciones lingüísticas propias de las prácticas académicas. Así, descubrimos que prácticamente no hay estrategias de este tipo en los libros de texto, lo cual nos lleva a plantear que no existen acciones metalingüísticas explícitas que faciliten el acceso de quienes estudian a la alfabetización académica por parte de las propuestas editoriales. En este sentido, y tal como sostiene Menéndez (1999, p. 102), el lector de los libros de texto se enfrenta con la imprecisión discursiva y debe superarla, en la medida en que él - o auxiliado por la persona docente - sería quien debería llenar el vacío que el discurso deja sin posibilidad de reponer. En efecto, los libros de texto no suelen propiciar 
ningún tipo de estrategia de facilitamiento que ayude al destinatario a comprender con mayor facilidad la complejidad polifónica. En otras palabras, no existen segmentos que comenten o propongan una reflexión sobre los mecanismos microdiscursivos (uso de comillas, inclusión de citas, presencia de conectores, nominalizaciones, etc.) ni tampoco se presentan preguntas que los retomen o que ahonden sobre la información que ofrecen o acerca de la controversia que desatan. Consideramos que sería necesario que el trabajo con el libro de texto se complemente con una lectura de tipo crítico y reflexivo, que implique formar juicios críticos, abordar problemas y analizar el discurso, para que el estudiante pueda lograr la autonomía en la interpretación y se forme como futuro sujeto de discurso académicocientífico, competencia que las personas ingresantes, al parecer, carecen.

\subsection{La relevancia del trabajo metalingüístico}

Atento a los resultados obtenidos en la experiencia descripta en los talleres de escritura académica, en las clases siguientes se puso en práctica una batería de actividades que buscaba promover la reflexión metalingüística. En este sentido, se abordó el texto de Ruiz mediante estrategias de facilitamiento centradas en la reflexión sobre ciertos fenómenos microdiscursivos que, originalmente, habían pasado inadvertidos por el estudiantado y que, por ende, se habían presentado como obstáculos para la comprensión del texto. Con tal propósito, se realizaron preguntas orales y escritas que proponían, por ejemplo, la reflexión acerca de los comentarios que el locutor realiza a través del uso de comillas (11) o la orientación argumentativa señalada por algunos conectores (12), entre otros fenómenos microdiscursivos. Paralelamente, se reflexionó y abordó la complejidad léxica que presenta el texto.

Por un lado, la principal dificultad hallada en torno a la interpretación de comillas o tipografía especial fue la descripta por Arnoux, Nogueira y Silvestri (2003, p. 5), la mayoría del estudiantado interpretaba "una indicación tipográfica más como reforzador que como distancia del enunciador respecto de lo enunciado". Para trabajar este aspecto, se releyó en profundidad el texto de Ruiz y luego se trabajó con una selección de diferentes textos argumentativos (periodísticos y académicos) con el fin de reconocer las diferentes funciones de las comillas. Así, los y las estudiantes comprobaron que las comillas pueden vehiculizar diversos tipos de comentarios y distinguieron algunas de sus funciones. Específicamente, en el texto de Ruiz, identificaron las comillas de tipo metalingüístico como las de "censura" y "censor"; las que introducen discurso directo, aunque no esté explícita la fuente del discurso 
citado (i.e. "formarse un juicio sobre una cosa, juzgar con sentido crítico"); o las llamadas "comillas de etiquetamiento" (García Negroni, Estrada y Mosqueda, 2006), que señalan el término acuñado por el autor del texto, en este caso "macdonalización" (11). ${ }^{9}$

Por otro lado, se hizo especial hincapié en el tipo y función de los conectores que aparecen en el texto de Ruiz, que tantos conflictos interpretativos trajeron. En (12), se exhiben algunas preguntas de facilitamiento en torno al conector "pero" del primer párrafo del texto que, como se recordará, no había sido interpretado exitosamente por una gran cantidad de estudiantes. Vale destacar que también se trabajó con otros conectores presentes en el texto, como "aunque", "en efecto", etcétera. Paralelamente, se reflexionó y abordó la complejidad léxica que presenta el texto ${ }^{10}$.

(11) ¿Qué función tienen las comillas que enmarcan estas expresiones: "censura", "censor", "formarse un juicio sobre una cosa, juzgar con sentido crítico" y "macdonalización"?

(12) ¿Qué tipo de conector es el que aparece subrayado en el fragmento? ¿Qué función cumplen en el texto? ¿Qué idea le permite introducir ese conector?

(13) "Se ha dicho que el discurso de la censura es un discurso que consiste, esencialmente, en amputar, suprimir, prohibir un cierto número o el conjunto de los hechos, ocultarlos, esconderlos. Razonar de esta forma es creer que, dada la abundancia de información, estamos en un universo donde los elementos son constantes. Pero hoy la censura no funciona mediante este principio".

Además, en el texto de Ruiz, como en los otros de tipo argumentativo trabajados en las clases siguientes, se examinaron los modos personales e impersonales de la presentación de conceptos y la postura del autor en la formulación de una denominación o terminología disciplinar. En este sentido, se abordaron las formas de personalización y despersonalización enunciativas (García Negroni, 2008) presentes en el discurso académico que, según manifestaron los y las estudiantes, desconocían. Dentro de las expresiones de personalización, en las sucesivas lecturas realizadas, se identificaron y caracterizaron la primera persona del singular (presentes en desinencias verbales y formas pronominales) y

\footnotetext{
9 García Negroni, Estrada y Mosqueda (2006) analizan el valor enunciativo de comillas y glosas en diversas disciplinas académicas, entendidas como manifestaciones metaenunciativas con las que el sujeto comenta su propia enunciación. Entre ellas, las autoras definen las comillas de etiquetamiento. Para profundizar sobre el tema, consultar García Negroni, Estrada y Mosqueda (2006).

10 No se presentan las actividades centradas en el léxico por cuestiones de espacio y porque este aspecto excede el objetivo de este trabajo.
} 
del plural (nosotros genérico, de modestia, de condescendencia); en cuanto a las formas de despersonalización (García Negroni, 2008), se caracterizaron las expresiones de infinitivo ("razonar así...", presente en el texto de Ruiz) las formas desagentivadas con pasiva con se (como "se sabe"), las nominalizaciones ("la investigación"), las frases nominales ("esta investigación", del texto de Ruiz"), la metonimia ("las conclusiones”), frases pasivas (“los resultados abordados").

También, se reflexionó especialmente sobre cómo puede emerger el discurso ajeno en un texto y qué expresiones lo introducen. A través de ejercicios de lectura y escritura, se caracterizaron las citas directas e indirectas y el discurso narrativizado ${ }^{11}$, y se distinguieron diversas formas despersonalizadas que pueden indicar la introducción de las citas, cuyo reconocimiento suele pasar inadvertido por parte de los lectores legos, como las formas desagentivadas con pasiva con se ("se ha dicho"); las nominalizaciones ("discusión" y "consenso"), las frases nominales (“la opinión”), la metonimia (“los métodos”).

Llegados a este punto cabe destacar que el trabajo centrado en la polifonía y la argumentación discursiva acarreó notables mejoras en el estudiantado. Por ejemplo, en el ejercicio final del taller, que versaba sobre la comprensión lectora de un texto argumentativo y la producción de un género académico breve, el $75 \%$ de la población estudiantil participante logró reconocer la hipótesis expresada por el autor del texto y el $90 \%$, la presencia de discurso ajeno. Como lo ha demostrado la presente experiencia, es indispensable llevar adelante un trabajo intenso sobre la materialidad discursiva de los textos especializados para promover la reflexión metalingüística, con el objetivo de contribuir a la constitución de estudiantes como sujetos de discurso académico.

\footnotetext{
11 Genette (1972 y 1989) define el discurso narrativizado como un modo de reproducción de discurso o de pensamiento en la narración literaria, el cual se caracteriza porque no se percibe citación ni reproducción del texto original. En este mismo sentido, Charaudeau (1997) advierte que, en el discurso narrativizado, lo dicho originalmente puede ser referido de modo que se integre totalmente hasta desaparecer en el decir de quien lo enuncia; se convierte así al locutor de origen en agente de un acto de decir. El discurso narrativizado puede detectarse porque está constituido por una expresión introductoria que contiene o no un verbo de decir, pero que está cargado siempre de una cierta subjetividad, y que no es seguido por la conjunción que, pues si no pertenecería al discurso indirecto. Moyano (2007) brinda el siguiente ejemplo: "El ministro cargó duro ayer contra la conducción del ente monetario" y así lo explica: "Allí cargar no es por sí solo un verbo de habla. Sin embargo en 'cargó duro contra' está refiriendo indudablemente a un discurso ajeno, donde esta expresión puede ser asociada a criticar" (Moyano, 2007, p. 38). Para profundizar sobre un análisis del discurso narrativizado en textos escolares, consultar Tosi (2016).
} 


\section{Palabras finales}

A lo largo del texto se exhibieron los resultados obtenidos en una experiencia didáctica llevada cabo en un taller de discurso académico destinado a ingresantes de un profesorado de Educación Primaria e Inicial en la Ciudad de Buenos Aires. El análisis corroboró que los principales problemas de comprensión del estudiantado se relacionan con la dimensión polifónica y argumentativa de los textos. Debido a que, en el nivel secundario, el material de estudio de los estudiantes lo constituyen los libros de texto- los cuales atenúan la complejidad discursiva con el propósito de allanar el proceso de aprendizaje- es posible afirmar que los ingresantes al nivel superior no están habituados a las características de la discursividad académica y de los textos especializados. Enfrentados con una configuración microdiscursiva diferente, el alumnado se topa con obstáculos discursivos que no sabe franquear. En efecto, al carecer de un entrenamiento en la lectura académica, no logra tener en cuenta, en la interpretación textual, los intersticios de significado, la dimensión argumentativa y polifónica de los textos, así como el componente subjetivo y crítico. Atento a ello y según lo desarrollado en estas páginas, resulta de vital importancia, por un lado, revisar la elaboración y elección de los libros de texto de nivel secundario por parte de editores y profesores, respectivamente, y, por el otro, atender al trabajo con la dimensión argumentativa-polifónica que se constituye como inherente a todo texto y que debe ser central para elaborar una pedagogía de la lectura en la formación docente.

La experiencia aquí descripta evidenció que la aplicación de las estrategias de facilitamiento, a las cuales definimos como aquellas que promueven la reflexión metalingüística y la desnaturalización de formulaciones cristalizadas, pueden contribuir a la formación de estudiantes como sujetos de discurso académico, pues habilitan la comprensión de ciertas formulaciones complejas. Si bien, la experiencia relatada se enmarcó en un taller específico de discurso académico, se estima que el trabajo de reflexión metalingüística sobre los textos se debe realizar en todas las materias de las carreras del nivel superior, porque cada disciplina presenta características discursivas propias.

Sin dudas, la problemática en cuanto a la comprensión lectora del estudiantado abre debates sobre el sentido y la finalidad del conocimiento, e invita a reflexionar acerca de los desafíos futuros en términos de la construcción de un proceso de aprendizaje plural y democrático que contemple la reflexión metalingüística y que realmente plantee la constitución de los y las estudiantes como sujetos de discurso académico. 


\section{Referencias}

Arnoux, Elvira, Di Stéfano, Mariana y Pereira, Cecilia. (2002). La lectura y la escritura en la universidad. Buenos Aires: Eudeba.

Arnoux, Elvira, Nogueira, Sylvia y Silvestri, Adriana. (2001). La escritura producida a partir de la lectura de textos polifónicos. Evaluación del desempeño de grupos con diferente entrenamiento lector previo. En María Cristina Martínez (Comp.), Aprendizaje de la argumentación razonada (pp. 49-77). Cali: Cátedra UNESCO y Universidad de Cali.

Arnoux, Elvira, Nogueira, Sylvia y Silvestri, Adriana. (2003). La construcción de representaciones enunciativas: el reconocimiento de voces en la comprensión de textos polifónicos. Signos, 35(51-52), 129-148.

Arnoux, Elvira, Nogueira, Sylvia y Silvestri, Adriana. (2006). Comprensión macroestructual y reformulación resuntiva de textos teóricos en estudiantes de institutos de formación de docentes primarios. Signos, 39(60), 9-30. Recuperado de http://www.scielo.cl/scielo.php?script=sci arttext\&pid=S0718-09342006000100001

Avendaño, Fernando y Perrone, Adriana. (2009). La didáctica del texto. Estrategias para comprender y producir textos en el aula. Rosario: Homo Sapiens.

Carlino, Paula. (2005). Escribir, leer y aprender en la universidad. Una introducción a la alfabetización académica. Buenos Aires: Fondo de Cultura Económica.

Charaudeau, Patrick. (1997). Le discours d'information mediatique. París: Nathan.

Chevallard, Yves. (1991). La transposición didáctica. Del saber sabio al saber enseñado. Buenos Aires: Aique.

de Souza Minayo, María Cecilia. (2009). La artesanía de la investigación cualitativa. Buenos Aires: Lugar.

Ducrot, Osvald. (2001). El decir y lo dicho. Barcelona: Paidós.

Duranti, Alessandro. (2000). Antropología lingüística. Cambridge University Press: Madrid.

García Negroni, María Marta y Hall, Beatriz. (2011). Prácticas discursivas escritas y orales en contexto universitario. Fragmentariedad y distorsiones enunciativas. En María Marta García Negroni (Coord.), Los discursos del saber. Prácticas discursivas y enunciación académica (pp. 191-222). Buenos Aires: Editoras del Calderón.

García Negroni, María Marta, Estrada, Andrea y Mosqueda, Ana. (2006). Polifonía y objetividad. Las no coincidencias del decir en el abstract científico. En Nilda Flawiá de Fernández y Silvia Israilev (Comps.), Hispanismo: discursos culturales, identidad y memoria (Vol. III, pp. 378-389).Tucumán: FFyL-UNT.

García Negroni, María Marta. (2005). Argumentación y polifonía en el discurso científicoacadémico. A propósito de ciertos conectores especializados en la trasgresión argumentativa. RASAL Lingüística, (1), 11-24. 
García Negroni, María Marta. (2008). Subjetividad y discurso científico-académico. Acerca de algunas manifestaciones de la subjetividad en el artículo de investigación en español. Signos, 41(66), 5-31.

Genette, Gerard. (1989). Nuevo discurso del relato. Madrid: Cátedra.

Genette, Gerard. (1972). Figures III. París: Seuil.

Gumperz, John (Ed.). (1982). Discourse strategies. New York: Cambridge University Press.

Hall, Beatriz y Marín, Marta. (2002). Marcas de argumentatividad en los textos de estudio: obstáculo para lectores. Actas del Congreso Internacional "La argumentación: Lingüística, Retórica, Lógica, Pedagogía”, Facultad de Filosofía y Letras de la Universidad Nacional de Buenos Aires.

Hall, Beatriz y Marín, Marta. (2003). Los puntos de incomprensión de la lectura en los textos de estudio. Lectura y Vida 24(1). Recuperado de http://www.lecturayvida.fahce.unlp.edu.ar/numeros/a24n1/24 01 Marin.pdf

Hall, Beatriz y Marín, Marta. (2011). El discurso académico-pedagógico: complejidad discursiva. En María Marta García Negroni (coord.) Los discursos del saber. Prácticas discursivas y enunciación académica (pp. 102-132). Buenos Aires: Editoras del Calderón.

Hall, Beatriz. (2007). La 'comunicación' científica en ámbitos académicos: otro enfoque. Revista Hologramática, Revista Académica de la Facultad de Ciencias Sociales de UNLZ, 2(7), 79-105.

Hall, Beatriz. (2008). Efectos de cientificidad y los modos de decir en el discurso académico en español. Línguas e Intrumentos Lingüísticos, (21), 53-75.

Johns, Ann y Swales, John. (2002). Literacy and disciplinary practices: Opening and closing perspectives. Journal of English for Academic Purposes, 1(1), 13-28.

Lerner, Delia. (2001). Leer y escribir en la escuela: lo real, lo posible y lo necesario. México: Fondo de Cultura Económica.

Litwin, Edith. (1997). Las configuraciones didácticas. Una nueva agenda para la enseñanza superior. Buenos Aires: Paidós.

Menéndez, Salvio. (1999). El discurso del libro de texto. Discurso y sociedad, 1(2), 55-68.

Moyano, Sara. (2007). El discurso narrativizado y las nominalizaciones citativas: dos formas solapadas de caracterizar la palabra ajena (Tesis de maestría). Universidad Nacional de La Plata, La Plata, Argentina.

Padilla, Constanza. (2010). La comunicación académica como construcción argumentativa: perspectivas de lectura y de escritura de estudiantes universitarios. En Víctor Castel y Liliana Cubo de Severino (Eds.), La renovación de la palabra en el bicentenario de la Argentina. Los colores de la mirada lingüística (pp. 963-970). Mendoza: UnCuyo. 
Parodi Sweis, Giovanni. (1999). Relaciones entre lectura y escritura: una perspectiva cognitiva discursiva. Bases teóricas y antecedentes empíricos. Valparaíso: Ediciones Universitarias de Valparaíso.

Pereira, Cecilia y Di Stéfano, Mariana. (Octubre, 2001). Textos argumentativos y textos expositivos. En Panel temático del Segundo Simposio Internacional de "Lectura y Vida", Buenos Aires, Argentina.

Solé, Isabel. (1999). Estrategias de lectura. Barcelona: GRAO.

Tosi, Carolina. (2015). Los modos de decir pedagógico en los libros de texto. Un análisis polifónico-argumentativo acerca de la especificidad genérica y sus efectos de sentido. Lengua $y$ Habla, 19, 126-148. Recuperado de http://erevistas.saber.ula.ve/index.php/lenguayhabla/article/view/6935

Tosi, Carolina. (2016). La voz de la mujer en los libros de texto argentinos: ausencias y discursos evocados. En Teresa Fernández Ulloa y Joanne Schmidt Morazzani (Eds.), Images of Women in Hispanic Culture (pp. 289-314). Bakersfield: Cambridge Scholars Publishing. 


\section{Anexo}

\section{La "macdonalización" del pensamiento}

Se ha dicho que el discurso de la censura es un discurso que consiste, esencialmente, en amputar, suprimir, prohibir un cierto número o el conjunto de los hechos, ocultarlos, esconderlos. Razonar de esta forma es creer que, dada la abundancia de información, estamos en un universo donde los elementos son constantes. Pero hoy la censura no funciona mediante este principio. En los sistemas, en apariencia democráticos, en los que nos encontramos inmersos existen pocos ejemplos de funcionamiento de la censura en los que, de una manera palmaria, se puedan ocultar, cortar, suprimir, prohibir los hechos. No se prohíbe en general a los periodistas decir lo que quieran; no se prohíbe a los periódicos en los países democráticos. La censura adopta otras modalidades de funcionamiento; se aplica sobre criterios diferentes. Hoy la censura no funciona suprimiendo, amputando, prohibiendo, cortando; actúa, por el contrario, por demasía, por acumulación. No permite asimilar ni pensar. Hay demasiado para consumir y, por lo tanto, no se percibe lo que falta. Actúa además en la concientización del discurso. El monopolio de la información unifica el mensaje y permite una "globalización" de la censura. Lo cierto es que la censura no ha desaparecido. Tan sólo ha cambiado de apariencia. [...]

La palabra "censura" provoca en cualquier individuo medianamente sensible, consciente de sus derechos y su libertad, un estremecimiento. Aunque tiene distintas acepciones, su connotación es tan fuerte que sólo asumimos parte de su significado, olvidando otros. En efecto, censurar es, en primer término, "formarse un juicio sobre una cosa, juzgar con sentido crítico", y solamente en una segunda acepción implica corrección o reprobación de una cosa. La distancia que va de reprobar a prohibir fue salvada rápidamente en la historia de la humanidad, y se transformó en una de sus cargas más pesadas.

La voz "censor" se relaciona etimológicamente con "censo" y ambas provienen del latín. En la Roma del siglo III, época de definiciones en el plano político, aparece el cargo de censor. Este magistrado estaba encargado del censo o inventario de los bienes sobre los cuales se aplicarían impuestos, y en consecuencia debía confeccionar una lista de los ciudadanos según su clase social. Los censores romanos también debían hacerse cargo de la ejecución de los trabajos públicos, establecían el presupuesto y finalmente podían tachar de infamia a los ciudadanos sospechosos de tener una moral dudosa. Teniendo en cuenta que sólo los ciudadanos de una cierta clase podían votar en asambleas, es fácil deducir que el censor de alguna manera eliminaba, fundado en principios éticos, a todo enemigo político. Claramente surge que, desde los albores, la censura es una práctica indefectiblemente ligada al poder político, a los intereses económicos de quienes detentan el poder y sólo en una tercera instancia involucra la reprobación -de hecho: prohibición- de lo considerado inmoral. Pero como la moral es sumamente elástica, ya que amplía o restringe sus límites según la época histórica, las prohibiciones tienen como rasgo característico la arbitrariedad más o menos furibunda de quien ejerce la censura. [...] 
La censura operó y opera en la Argentina como una forma de preservar valores fijos, estratificados en la sociedad o en los grupos dominantes, sean estos religiosos, económicos o políticos. El objetivo que persigue la censura es que esos valores no cambien, no se modifiquen, y que el orden impuesto persista. [...]

Quizá en nuestro país sea necesario descubrir no tanto el por qué, sino el cómo para modificar esta realidad. Este es un mundo peligrosamente parecido al de los mudos y los sordos, porque hay una tendencia al mensaje único. Que se debe a la concentración del poder que se da en la economía, pero también en la cultura; cada vez es más difícil el discurso disidente. En esta "macdonalización" del pensamiento, la censura es un hecho natural.

La idiotez y la hipocresía capitalistas se han tornado verdad absoluta en el marco de este nuevo orden mundial, donde los burócratas se hacen empresarios y los censores se vuelven campeones de la libertad de expresión. Esta investigación intenta ser, como diría Cesare Pavese, "la auscultación de una perplejidad" Ilamada Argentina. La pesada tarea de vivir en una sociedad que se funde entre los silencios cómplices y la intolerancia.

Prólogo escrito por Néstor Ruiz al libro Una historia de la censura: violencia y proscripción en la Argentina del siglo XX, de Fernando Ferreira (Ed. Norma, Buenos Aires, 2000).

1) ¿Cuál es la hipótesis propuesta por el autor?

2) ¿Cómo defiende el autor su hipótesis? 